\title{
NV center emission in a substrate free low index environment
}

F. A. Inam, M. D. W. Grogan, M. Rollings, T. Gaebel, S. Castelletto, et al.

F. A. Inam, M. D. W. Grogan, M. Rollings, T. Gaebel, S. Castelletto, J. M. Say, C. Bradac, T. A. Birks, W. J. Wadsworth, J. R. Rabeau, M. J. Steel, "NV center emission in a substrate free low index environment," Proc. SPIE 8635, Advances in Photonics of Quantum Computing, Memory, and Communication VI, 86350F (29 March 2013); doi: 10.1117/12.2003493

SPIE. Event: SPIE OPTO, 2013, San Francisco, California, United States 


\title{
NV center emission in a substrate free low index environment
}

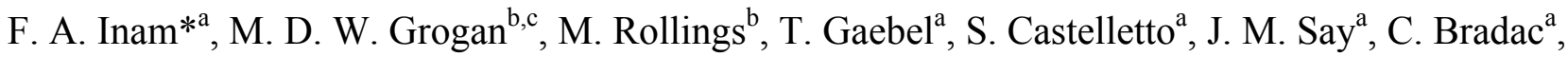 \\ T. A. Birks ${ }^{\mathrm{a}}$, W. J. Wadsworth ${ }^{\mathrm{a}}$, J. R. Rabeau ${ }^{\mathrm{a}}$, and M. J. Steel ${ }^{\mathrm{a}}$ \\ ${ }^{a}$ MQ Photonics Research Centre, Department of Physics and Astronomy, Macquarie University, North Ryde 2109,

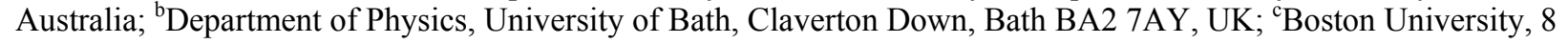 \\ Saint Mary's St., Boston, MA 02215, USA
}

\begin{abstract}
With in-built advantages (high quantum efficiency and room temperature photostability ${ }^{1}$ ) for deployment in quantum technologies as a bright on-demand source of single photons, the nitrogen vacancy (NV) center is the most widely studied optical defect in diamond. Despite significant success in controlling its spontaneous emission ${ }^{2}$, the fundamental understanding of its photo-physics in various environments and host material remains incomplete. Studying NV photoemission from nanodiamonds on a glass substrate, we recently pointed out a disparity between the measured and calculated decay rates (assuming near unity quantum efficiency) ${ }^{3}$. This indicates the presence of some strong nonradiative influences from factors most likely intrinsic to nanodiamond itself. To obtain a clearer picture of the NV emission, here we remove the substrate contributions to the decay rates by embedding our nanodiamonds inside silica aerogel, a substrate-free environment of effective index $n \sim 1.05$.

Nanodiamond doped aerogel samples were fabricated using the "two-step" process ${ }^{4}$. Time-resolved fluorescence measurement on $\sim 20$ centers for both coverslip and aerogel configurations, showed an increase in the mean lifetime $(\sim 37 \%)$ and narrowing of the distribution width $(\sim 40 \%)$ in the aerogel environment, which we associate with the absence of a air/cover-glass interface near the radiating dipoles ${ }^{3}$. Finite difference time domain (FDTD) calculations showed the strong influence of the irregular nanodiamond geometry on the remaining distribution width. Finally a comparison between measurements and calculations provides an estimate of the quantum efficiency of the nanodiamond $\mathrm{NV}$ emitters as $\sim 0.7$. This value is apparently consistent with recent reports concerning the oscillation of the NV center between negative and neutral charge states ${ }^{5}$.
\end{abstract}

Keywords: Single photon source, spontaneous emission, aerogel environment, single center emission, diamond NV center, lifetime distribution, diamond crystal geometry, quantum efficiency

\section{INTRODUCTION}

Scalable realizations in the emerging field of quantum technologies like quantum cryptography and quantum computation demand bright solid state optical sources emitting single photons on-demand ${ }^{6}$. Diamond NV center has emerged as one of the most promising solid state candidates, owing to its room temperature photo-stability and high quantum efficiency ${ }^{7,8}$. As a result, a number of approaches have been successfully employed to manipulate the rate of spontaneous emission of NV dipole emitters by altering the local electromagnetic environment ${ }^{9-12}$. In-spite of achieving significant progress in enhancing the rate of its spontaneous emission, the fundamental understanding of the process of its emission is far from exhaustive. Our previous study on the emission from these NV centers inside nanodiamond crystals on glass substrate has pointed out an existing large disparity between the observed decay rates and their calculations, assuming near quantum efficiency ${ }^{3}$. This discrepancy indicates the presence of strong non-radiative factors significantly influencing the decay rates. These factors may include the quenching from the outer graphite shell ${ }^{13}$, photoinduced charge conversion between the $\mathrm{NV}^{-}$and $\mathrm{NV}^{0}$ charge states ${ }^{14}$, surface chemistry ${ }^{15}$ and other factors such as spectral diffusion, largely related to $\mathrm{NV}$ emission within the nanodiamond lattice. Therefore a more complete understanding of its intrinsic emission properties is required for the successful deployment of these centers for bright single photon sources.

To achieve a better understanding of the process of spontaneous emission from these NV centers, in this work we aim to isolate the external contributions due to the substrate on the emission rate. Under the standard scheme of study where the 
nanodiamonds are placed on a glass substrate, the surrounding air-glass interface is known to influence the emission rates of NV dipole emitters ${ }^{3}$. Also a recent study at cryogenic temperatures has shown that the phonon sideband of NV emission spectrum can be drastically altered by just changing the substrate ${ }^{15}$. Therefore an ideal scenario to fundamentally study the emission from these centers would be to hold them in air, in an environment of index very close to unity. Since our $50 \mathrm{~nm}$ sized nanodiamonds crystals are non-spherical ${ }^{3}$ and too small to be effectively trapped using optical tweezers, we studied the spontaneous emission from these nanodiamond NV centers by placing the diamonds inside silica aerogel where the effective index of the environment is around 1.05.

Our work is therefore two-fold relevant, first from the material prospective, we were able to synthesize good quality nanodiamond doped aerogel samples, where single centre emission properties are preserved without being drastically altered by the background fluorescence from the synthesis process, and more importantly from the point that we were able to separate the influence of the substrate-studying the emission largely under the influence of the intrinsic local nano-environment of the host crystal itself. Second, by doing a comparative study between measured and theoretical rates for the two environments-glass coverslip and aerogel we were able to estimate the quantum efficiency of these nanodiamond NV emitters, thereby adding significant value to the present understanding of their spontaneous emission.

\section{MEASUREMENTS}

Silica aerogel is a low density, porous material made up of interconnected nanoscopic strands of glass particles contributing up to $10 \%$ of the macroscopic volume of the sample ${ }^{16}$. This sub-wavelength structured network of glass particles causes Rayleigh-like scattering giving aerogel an opalescent appearance. For a 5\% volume of glass particles the refractive index of the sample is generally around 1.03-1.05 and can be altered slightly by varying the growth parameters. The dimensions of the aerogel structure is around $1-5 \mathrm{~nm}$ for the silica glass and around $50 \mathrm{~nm}$ for the airfilled pores making it feasible for the trapping of many nanoparticles ${ }^{17}$. Utilizing this aspect of trapping nanoparticles inside the air-filled pores, we fabricated nanodiamonds (average size $\sim 54 \mathrm{~nm}$ ) in a doped aerogel sample following the "two-step" growth process employed for doping of similar size gold particles ${ }^{4}$.

To characterize the structure of diamond doped aerogels, we performed transmission electron microscopy (TEM) measurement on a thin $\sim 70 \mathrm{~nm}$ cross-section of the sample. Some of the TEM images displayed isolated diamond crystals embedded in the silica glass matrix of the aerogel.

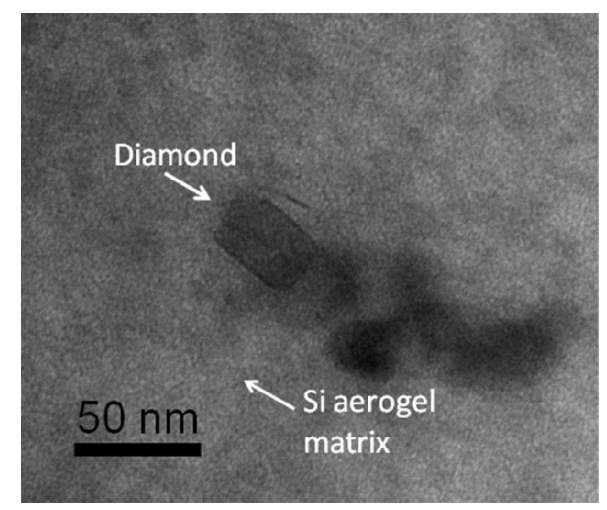

Figure 1. TEM image showing an isolated diamond crystal embedded inside the silica aerogel matrix.

The diamond doped aerogel sample was then imaged under a confocal microscope. Fluorescence from both the coverslip and aerogel samples were collected using a $532 \mathrm{~nm}, 100 \mathrm{~mW}$ continuous-wave laser with around $200 \mu \mathrm{W}$ power incident on the sample ${ }^{3}$. The aerogel sample was attached to the surface of a coverslip and the laser was focused from the coverslip's other surface to planes $\sim 40-90 \mu \mathrm{m}$ inside the aerogel using a $100 \times$ infinity-corrected oil immersion objective lens with a numerical aperture of 1.3. A spectrometer was used to characterize the luminescence and a Hanbury-Brown 
and Twiss (HBT) interferometer was used to perform the single photon statistics. Nanodiamonds hosting only single centers (having second order intensity correlation function $\left.g^{(2)}(0)<0.5\right)$ were studied with $N_{c}=28$ on coverslip and $N_{a}=20$ in aerogel. The lifetime of these single centers was determined using time resolved fluorescence measurements using a $532 \mathrm{~nm}$ pulsed laser.

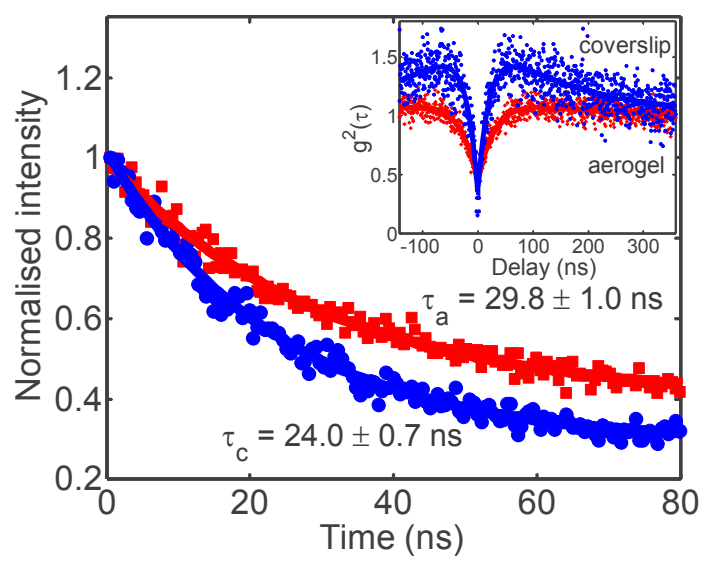

Figure 2. Time resolved normalized fluorescence decay. Red curve: aerogel, and blue curve: coverslip. Inset: Second order auto-correlation curve.

From the collected data we found the mean lifetime for nanodiamonds on coverslip $\tau_{c}=22.9 \pm 1.6 \mathrm{~ns}$ and that for diamonds in aerogel as $\tau_{a}=31.3 \pm 1.4 \mathrm{~ns}$. The corresponding distribution widths were $\Delta \tau_{c}=8.3 \pm 3.5 \mathrm{~ns}$ for coverslip and $\Delta \tau_{a}=6.3 \pm 2.8$ on aerogel. The error in the width corresponds to variance of the variance.

While the mean lifetime increases from coverslip to aerogel, there is a reduction in the relative width $\Delta \tau / \tau$. This change in relative width can be expected due to the removal of the interference contributions to the electric field from the air-glass interface. After isolating the contributions from the substrate, the remaining influences on the emission now can arise solely from factors intrinsic to the NV emission within the nanodiamond crystal itself: shape, spectral diffusion or strain which would be contributing to the remaining width.
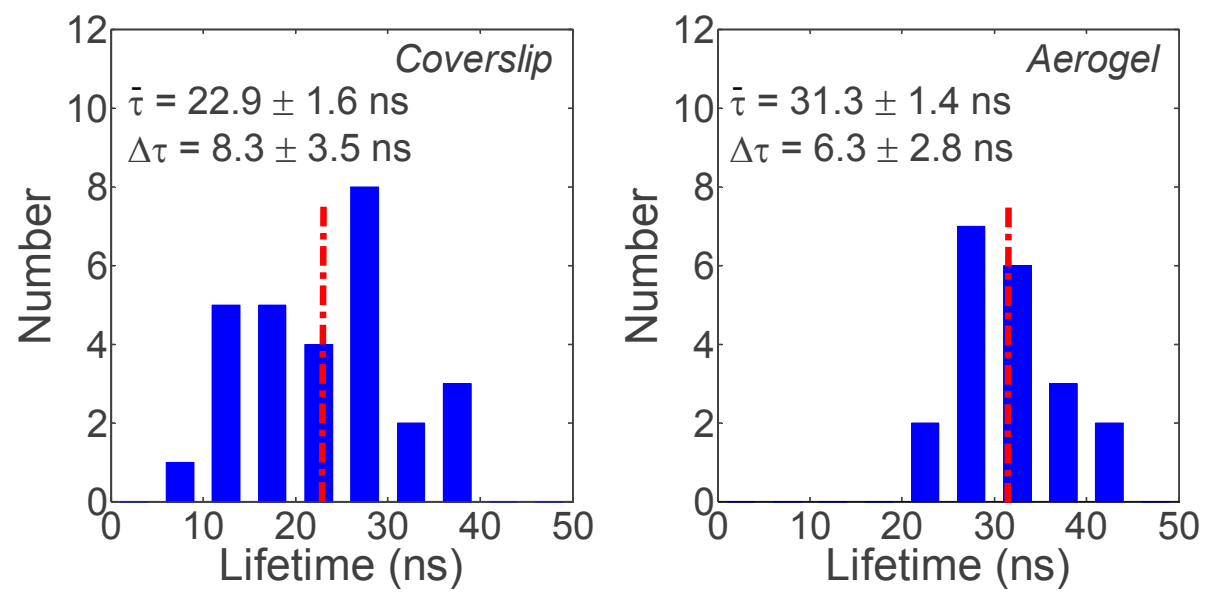

Figure 3. Experimentally measured distribution of NV centers lifetimes a) on coverslip and b) inside aerogel. 


\section{CALCUlations}

As majority of the decay from the diamond NV centers is known to be radiative, ${ }^{7,8}$, we performed finite-difference timedomain calculations for the two measured schemes of nanodiamonds on coverslip and inside aerogel to get a better picture of the influence of environment on the emission lifetimes of these NV emitters. By comparing the power radiated by an oscillating dipole inside a nanodiamond sphere with the power radiated by the same dipole in bulk diamond material, we calculated the radiative spontaneous emission rates relative to bulk, $\gamma_{i}=P_{i} / P_{b}$ for the respective schemes of coverslip/aerogel. As our nanodiamonds are known to be very irregular in shape ${ }^{3}$ we performed calculations for three different geometrical representations: sphere, cube and trapezoid, each of dimension $54 \mathrm{~nm}$ (average size of our nanodiamond crystal). The observed lifetime distribution should lie within the three geometries, somehow closer to the intermediate scheme of a trapezoid. We build our distribution of lifetimes by performing calculations over an ensemble of 250 random dipole locations inside the diamond crystal. To represent the aerogel environment, calculations were done for a medium of effective index 1.05 and a $5 \mathrm{~nm}$ shell of silica glass enclosing the diamond crysta ${ }^{18}$.

Calculations showed that the difference between the results of the two aerogel representations is minimal; indicating that the measured lifetimes would not have been significantly influenced by the random porous structures of silica glass particles $(\sim 1-5 \mathrm{~nm})$ around the diamond crystal. Figure 4 therefore only presents the aerogel results corresponding to the average index representation of the aerogel.

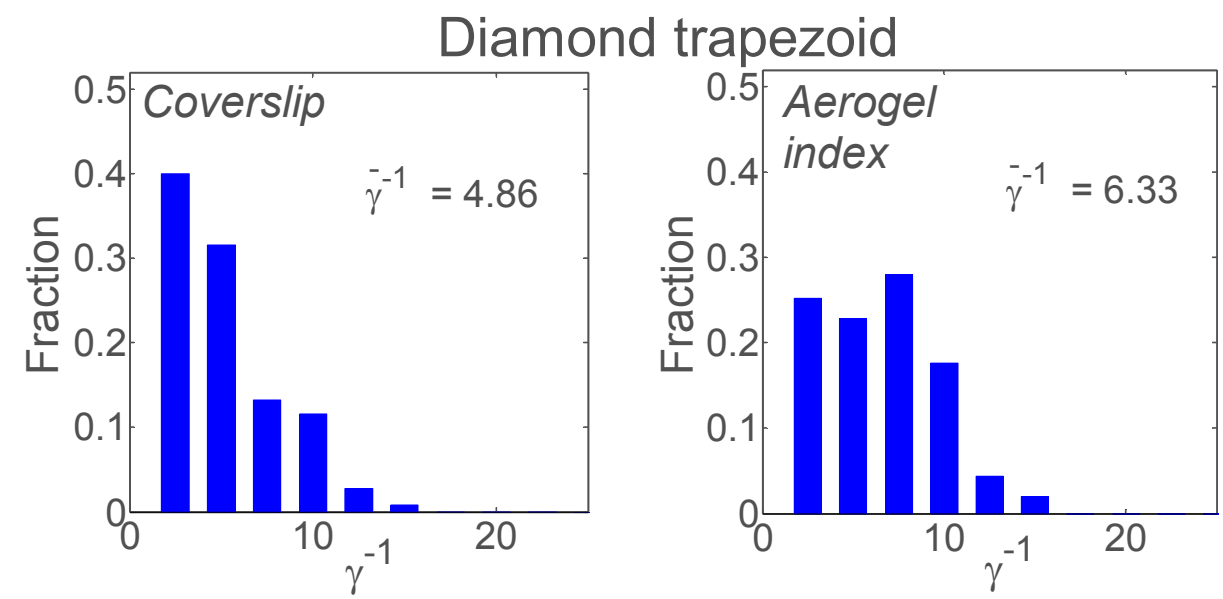

Figure 4. FDTD caculated normalzed radiative lifetime distribution $\gamma^{-1}$ for NV emission inside a trapeoidal diamond crystal of size $54 \mathrm{~nm}$ placed on coveslip and aerogel index medium.

For a dipole emitting inside a sub-wavelength dielectric sphere the emission rate is known to be almost independent of the dipole locations and orientations ${ }^{19}$. Our calculations also displayed a very narrow distribution width for NV centers embedded inside the high dielectric constant diamond sphere. For the case of cube or trapezoid the dipoles emitting near the edges experience distinct electromagnetic environments for distinct dipole polarizations, giving rise to a broader distribution. Further the large gradient of refractive index at the edges results in large electric fields around, thereby increasing the power radiated (i.e. higher decay rates $\gamma$ ) by the surrounding dipoles. As a result the mean lifetime, $\bar{\gamma}^{-1}$ is smaller compared to that on sphere. Though there remain significant differences in the calculated results for sphere and the other two geometries, the relative change in the lifetimes from coverslip to aerogel index is similar for all of them, also corresponding to the change in the measured lifetimes. As our nanodiamonds are known to be pretty irregular in geometry ${ }^{3}$, we believe that the trapezoid, possessing a convex geometry intermediate to the two extremes of sphere and cube is the best representative for them. Therefore in the rest of the paper, we only consider our calculations corresponding to the trapezoidal geometry. 


\section{QUANTUM EFFICIENCY}

In the literature, there exists a large discrepancy on the measured quantum efficiency of the diamond NV centers, with reported values ranging from 0.7 to $1.0 .^{5,7,8,20}$ In this work we measured the decay rates for two different configurations of nanodiamonds on coverslip and inside aerogel. This allows us to make new estimations for the quantum efficiency of the nanodiamond NV center.

The estimation of the quantum efficiency requires a comparison of the calculated to observed rates taking into account the contributions of the non-radiative decay. Considering the non-radiative decay rates to be same for the bulk and the nanodiamond, we extract the value of the quantum efficiency that reconciles the measured decay rates and calculated emission rates, finding $\eta=0.75 \pm 0.05$ and $\eta=0.62 \pm 0.08$ for the aerogel and coverslip, respectively.

The assumption of the same non-radiative decay for the bulk and the nanodiamond is build around that fact that the most dominant non-radiative contribution comes from the intersystem crossing level or the photo-conversion between the $\mathrm{NV}^{-}$ and $\mathrm{NV}^{0}$ charge states. ${ }^{5}$ Figure 5 shows contours of the expected total life time enhancement factor $\beta\left(\beta_{i} \equiv \tau_{i} / \tau^{\infty}\right)$ as a function of the quantum efficiency $\eta$ and radiative enhancement $\gamma$. The red and blue dots in Figure 5 correspond to the measured lifetime $\beta_{i}$ and calculated enhancement $\gamma_{i}$ for the aerogel and coverslip cases, respectively.

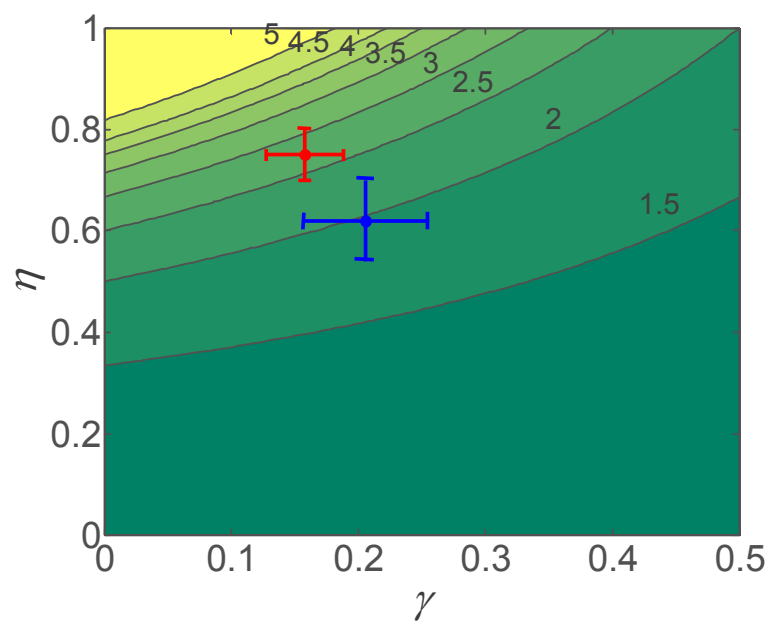

Figure 5. Contours of total lifetime enhancement $\beta$ as a function of quantum efficiency $\eta$ and radiative enhancement $\gamma$. Blue dot: coverslip, Red: aerogel. The error-bars indicate one standard deviation uncertainties.

\section{CONCLUSIONS}

In this work we isolated the effect of the local nano-environment of the host diamond crystal on the emission rates of the $\mathrm{NV}$ emitters by embedding our nanodiamonds in an aerogel lattice where the effective refractive index of the environment is very close to unity. We identified that the host crystal morphology plays a significant role in modifying the rate of spontaneous emission, and should be taken into consideration while performing radiative emission calculations for nanodiamond NV centers. A comparison between measurements and calculations resulted in the estimation of the quantum efficiency of the nanodiamond $\mathrm{NV}$ emitters as $\sim 0.7$. This value is apparently consistent with recent reports concerning the oscillation of the NV center between negative and neutral charge states. ${ }^{5}$ In conclusion, our work provides a better understanding of the primary factors influencing the emission of these diamond NV centers, adding relevant information for their future deployment in nanoscale applications. 


\section{ACKNOWLEDGEMENT}

The work at Macquarie was produced with the assistance of the Australian Research Council under the ARC Center of Excellence Program (EQuS), project number CE110001013. Calculations for the work were performed using the NCI computational facility. F. A. Inam thanks Debra Birch for TEM measurements performed at the Macquarie University Microscopy Unit. The work at Bath was funded by EPSRC grant EP/F018622/1 "Aerogels in fibre optics". T. A. Birks thanks the Leverhulme Trust for a Research Fellowship.

\section{REFERENCES}

[1] Kurtsiefer, C., Mayer, S., Zarda, P., and Weinfurter, H., "Stable solid-state source of single photons," Physical Review Letters, 85, 290-293, (2000).

[2] Aharonovich, I., Greentree, A. D., and Prawer, S., "Diamond photonics," Nat Photon, 5, 397-405, (2011).

[3] Inam, F. A. and Et Al., "Modification of spontaneous emission from nanodiamond colour centres on a structured surface," New Journal of Physics, 13, 073012, (2011).

[4] Grogan, M. D. W., Heck, S. C., Xiao, L. M., England, R., Maier, S. A., and Birks, T. A., "Control of nanoparticle aggregation in aerogel hosts," Journal of Non-Crystalline Solids, 358, 241-245, (2012).

[5] Waldherr, G., Beck, J., Steiner, M., Neumann, P., Gali, A., Frauenheim, T., Jelezko, F., and Wrachtrup, J., "Dark States of Single Nitrogen-Vacancy Centers in Diamond Unraveled by Single Shot NMR," Physical Review Letters, 106, 157601, (2011).

[6] Scheel, S., "Single-photon sources-an introduction," Journal of Modern Optics, 56, 141-160, (2009).

[7] Schietinger, S., Barth, M., Alchele, T., and Benson, O., "Plasmon-Enhanced Single Photon Emission from a Nanoassembled Metal-Diamond Hybrid Structure at Room Temperature," Nano Letters, 9, 1694-1698, (2009).

[8] Rittweger, E., Han, K. Y., Irvine, S. E., Eggeling, C., and Hell, S. W., "STED microscopy reveals crystal colour centres with nanometric resolution," Nat Photon, 3, 144-147, (2009).

[9] Hausmann, B. J. M., Shields, B., Quan, Q., Maletinsky, P., Mccutcheon, M., Choy, J. T., Babinec, T. M., Kubanek, A., Yacoby, A., Lukin, M. D., and LončAr, M., "Integrated Diamond Networks for Quantum Nanophotonics," Nano Letters, 12, 1578-1582, (2012).

[10] Park, Y. S., Cook, A. K., and Wang, H. L., "Cavity QED with diamond nanocrystals and silica microspheres," Nano Letters, 6, 2075-2079, (2006).

[11] Wolters, J., Schell, A. W., Kewes, G., Nusse, N., Schoengen, M., Doscher, H., Hannappel, T., Lochel, B., Barth, M., and Benson, O., "Enhancement of the Zero Phonon Line emission from a Single NV-Center in a Nanodiamond via Coupling to a Photonic Crystal Cavity," Applied Physics Letters, 97, 141108/1-3, (2010).

[12] Choy, J. T., Hausmann, B. J. M., Babinec, T. M., Bulu, I., Khan, M., Maletinsky, P., Yacoby, A., and Loncar, M., "Enhanced single-photon emission from a diamond-silver aperture," Nat Photon, 5, 738-743, (2011).

[13] Smith, B. R., Gruber, D., and Plakhotnik, T., "The effects of surface oxidation on luminescence of nano diamonds," Diamond and Related Materials, 19, 314-318, (2009).

[14] Siyushev, P., Pinto, H., Gali, A., Jelezko, F., and Wrachtrup, J., "Low Temperature Studies of Charge Dynamics of Nitrogen-Vacancy Defect in Diamond," DOI: arXiv:1204.4898v1, (2012).

[15] Zhao, H.-Q., Fujiwara, M., and Takeuchi, S., "Suppression of fluorescence phonon sideband from nitrogen vacancy centers in diamond nanocrystals by substrate effect," Opt. Express, 20, 15628-15635, (2012).

[16] Pajonk, G. M., "Transparent silica aerogels," Journal of Non-Crystalline Solids, 225, 307-314, (1998).

[17] Morris, C. A., Anderson, M. L., Stroud, R. M., Merzbacher, C. I., and Rolison, D. R., "Silica Sol as a Nanoglue: Flexible Synthesis of Composite Aerogels," Science, 284, 622-624, (1999).

[18] Grogan, M. D. W., Heck, S. C., Hood, K. M., Maier, S. A., and Birks, T. A., "Structure of plasmonic aerogel and the breakdown of the effective medium approximation," Optics Letters, 36, 358-360, (2011).

[19] Chew, H., "Radiation and lifetimes of atoms inside dielectric particles," Physical Review A, 38, 3410, (1988).

[20] Gruber, A., Dräbenstedt, A., Tietz, C., Fleury, L., Wrachtrup, J., and Borczyskowski, C. V., "Scanning Confocal Optical Microscopy and Magnetic Resonance on Single Defect Centers," Science, 276, 2012-2014, (1997). 